\title{
Allergie und Asthma: Labormedizinische Herausforderungen für eine Molekulare Diagnostik
}

\author{
H. Renz'
}

Allergie und Asthma haben als Volkskrankheiten einen erheblich medizinischen und sozio-ökonomischen Impact. Dieser ist charakterisiert durch folgende Fakten:

- Zwischen 24-32 Millionen Menschen in Deutschland leiden an einer Allergie.

- Die Tendenz ist steigend.

- Die Ursachen liegen in einer multidirektionalen Interaktion zwischen genetischer Disposition, immunologischer Fehlregulation und Umweltbedingungen (,westliche Lebensweise").

- Die gegenwärtig verfügbare Therapie ist ausschließlich symptomatisch. Eine Kausaltherapie steht nicht zur Verfügung.

- Sekundär- und Tertiär-Prävention ist nur bedingt erfolgreich. Eine Primärprävention steht nicht zur Verfügung.

- Die verfügbare in-vitro Diagnostik ist insuffizient. Sie beschränkt sich im wesentlichen auf die Messung von allergenspezifischen IgE-Antikörpern. Eine molekulare Diagnostik vom Genotyp zum Phänotyp ist dringend erforderlich. Marker zur Prädiktion stehen nicht zur Verfügung.

- Allergie- und Asthma-Behandlungen kosten ca. 10-15 Mrd. DM pro Jahr (direkte und indirekte Kosten).

In den letzten Jahren konnten erhebliche Fortschritte im immunologischen Verständnis der zugrunde liegenden Fehlregulation bei Allergie und Asthma erzielt werden. Ziel unserer Arbeitsgruppe ist hierbei über ein besseres Verständnis der Pathogenese von Allergie und Asthma zu einer Optimierung von Diagnostik und Therapiestrategien zu gelangen. Hierzu sind zum einen eine Reihe von Tiermodellen (Maus) entwickelt worden, in denen wesentliche Grundzüge der immunologischen Fehlregulation in-vivo induziert werden konnten. Diese Tiermodelle eignen sich zur Untersuchung der Pathogenese. Hierbei steht die Induktion und Perpetuation einer fehlgeleiteten T-Zell Immunantwort im Mittelpunkt. Diese Zellen sind die einzigen Zellen des Immunsystems, die antigen-spezifische Funktionen entfalten können. Ferner werden diese Modelle angewandt, um neue immunmodulatorische Stategien ebenfalls unter in-vivo Bedingungen zu evaluieren. Ein An-

Kurzfassung eines Vortrags, gehalten anläßlich der Verleihung des Gabor Szasz-Preises am 3. Oktober 1999 in Regensburg (s. Bericht in J Lab Med 1999;23: 722)

'Adresse: Priv.-Doz. Dr. med. Harald Renz. Abt. fûr Klinische Chemie und Zentrallaboratorium, Philipps Universităt Marburg. Fax +49-6421-28-65594 satzpunkt ist hierbei die Intervention auf der Ebene der fehlgeleiteten Zytokinproduktion durch Applikation von Zytokinantagonisten, löslichen Rezeptoren etc. Parallel hierzu ist die Ausbildung einer immunologischen Toleranz durch spezifische Immuntherapie als Intervention auf der Ebene der antigenspezifischen TZellen im Mittelpunkt. Da in diesem Zusammenhang auf Details nicht eingegangen werden kann, wird auf aktuelle Übersichtsarbeiten verwiesen (1-5).

Im Gegensatz zu anderen chronischen Entzündungserkrankungen zeichnen sich Allergie und Asthma durch die präferenzielle Aktivierung einer besonderen T-Zell Effektorpopulation aus, die als TH-2 Immunantwort definiert ist. Das charakteristische einer TH-2 Immunantwort ist ihre regulatorische Kompetenz auf eosinophile Granulozyten, Mastzellen und IgE-Synthese. Damit stellt diese Form der T-Zell Effektorantwort eine Schlüsselstelle im allergischen Geschehen dar. Auslöser bzw. Initiator der Immunantwort ist der Kontakt mit an und für sich harmlosen Umweltantigenen. Die normale Immunantwort gegenüber Hausstaubmilben, Pollen, Nahrungsmitteln etc. ist eine immunologische Toleranz. Beim Allergiker hingegen ist das Immunsystem nicht in der Lage, eine Toleranzantwort aktiv zu formen, sondern es kommt zur Ausbildung einer fehlgeleiteten TH-2 Effektorantwort. Die Entwicklung dieses regulatorischen Konzepts trägt zur Entwicklung von spezifischen Interventionsstrategien bei, die gezielt in das Immungeschehen eingreifen sollen. Hierbei soll die heraufregulierte TH-2 Antwort direkt oder indirekt antagonisiert werden. Dieses Ziel wird mit der Entwicklung von Zytokinantagonisten, löslichen Zytokinrezeptoren, monoklonalen Antikörpern etc. verfolgt. Einige dieser Substanzen, wie z.B. ein rekombinanter löslicher IL-4 Rezeptor und monoklonale Antikörper gegen IL-5 sind bereits in der klinischen Erprobung. Ein weiterer Ansatz ist die Verhinderung der immunologischen Fehlregulation durch frühzeitige Toleranzinduktion. Hier werden unter dem Stichwort einer „präventiven allergischen Impfung“ eine Reihe von neuen Ansätzen evaluiert: Mukosale Toleranzinduktion, Behandlung mit immunstimulatorischen Sequenzen aus bakterieller DNA [CpG Oligonukleotide], präventive Immuntherapie mit hohen Allergendosen und andere mehr.

Um die Ausbildung von Allergie und Asthma primär zu verhindern, ist es erforderlich, die initialen Schritte der Immunaktivierung zu verstehen. Wann im Leben und durch welche molekularen Ereignisse wird die immunologische Fehlregulation in Gang gesetzt? 
Dieses Forschungsfeld gewinnt in allerjüngster Zeit an Dynamik, da eine Viclzahl von Erkenntnissen belegen, daß die materno-fetalen Interaktionen offensichtlich deutlich komplexer sind als bisher angenommen. Hicr zeichnet sich ein bahnender Einfluß auf die Allergieentstehung ab. Es gibt Hinweise, die zeigen, daß das maternale Immunsystem die Ausbildung der fetalen Immunität ab etwa dem zweiten Trimenon der Schwangerschaft funktionell beeinflußt. Gleichzeitig kommit es während der Schwangerschaft zu einer lokalen immunologischen Umorganisation, womit die Abstoßung des fetalen "Allotransplantats" und damit einen Abort verhindert werden soll.

Diese Erkenntnisse münden auch in eine Weiterentwicklung der Diagnostik. Die molekulare Charakterisierung von Hauptallergenen erlaubt heute über die Herstellung von rekombinanten Majorallergenen eine valide quantitative Bestimmung von allergenspezifischen IgE-Antikörpern. Die Messung spezifischer IgEAntikörper ist nach wie vor die Domäne der in viț̣o Allergiediagnostik. Patienten unter spezifischer Immuntherapie bedürfen eines intensiven immunologischen Monitorings. Hier gibt es erhebliche Defizite, da bis heute keine Marker zur Verfügung stehen, die den Erfolg einer solchen Therapie sicher anzeigen können. Ferner ist ein immunologisches Monitoring erforderlich, um Patienten zu selektionieren, die von einer spezifischen immunologischen Intervention profitieren. $\mathrm{Da}$ diese Therapiestrategien aufwendig, zeit- und kostenintensiv sind, ist eine Weiterentwicklung dringend erforderlich. Ferner entwickelt sich das Umweltmonitoring zu einem unverzichtbaren Bestandteil einer modernen Allergiediagnostik. Die Verfügbarkeit monoklonaler Antikörper, gerichtet gegen Majorallergene erlaubt heute die Quantifizierung von Allergenen in der Umwelt des Patienten. Dieser neue Aspekt der Allergiediagnostik wird in den nächsten Jahren sehr an Bedeutung gewinnen.

Die Entwicklung und Empfehlung von Präventivstrategien setzt eine sichere Prädiktion für die Entwicklung von Allergie und Asthma voraus. Viel Enthusiasmus ist mit der Messung von Gesamt-IgE im Nabelschnurblut verbunden gewesen, allerdings haben große prospektive Kohortenstudien belegt, daß dieser Parameter kein geeigneter Prädiktionsmarker für ein Populationsscreening darstellt. In jüngster Zeit eröff- net die Molekulargenetik eine neue Perspektive. Daß Allergie und Asthma eine genetische Komponente haben, steht außer Zweifel, jedoch wird ebenso deutlich, daß es sich hierbei nicht um eine monogene $\mathrm{Er}$ krankung handelt, sondern daß Major- und Minorgene komplex ineinander greifen. Dieses Bild wird noch dadurch kompliziert, daß offensichtlich verschiedene Phänotypen wie z.B. IgE-Produktion, bronchiale Hyperreagibilität, Ausbildung bestimmter Entzündungsformen, organspezifische Manifestationen wie Neurodermitis und Asthma mit Mutationen an unterschiedliche Genloci gekoppelt sind. Ferner zeigen Untersuchungen in verschiedenen ethnischen Populationen wiederum unterschiedlich genetische Koppelungen. Dieses komplexe Bild kann möglicherweise durch den Einsatz neuerer Technologien für eine molekulargenetische Risikodiagnostik genutzt werden. Hier eröffnet die molekulargenetische Diagnostik mittels Chiptechnologien eine Perspektive.

Hieraus wird deutlich, daß die Laboratoriumsmedizin erheblich von einer kliniknahen Grundlagenforschung profitiert, die die immunologische Fehlregulation allergischer Erkrankungen analysiert, die genetische Komponente versucht zu identifizieren und hieraus neben diagnostischen insbesondere auch moderne therapeutische Konzepte entwickelt. Die enge Verzahnung zwischen kliniknaher Grundlagenforschung, Labordiagnostik und Klinik erfordert ein interdisziplinäres Netzwerk vor Ort, um zukunftsorientiert die Probleme und Herausforderungen dieser Volkskrankheiten anzugehen.

\section{Literatur}

1. Herz U, Bunikowski R, Renz H. Role of T Cells in Atopic Dermatitis. Int Arch Allergy Immunol 1998;115:179-90.

2. Meissner N, Kochs S, Coutelle J, Kussebi F, Baumgarten C, Löwenstein H, Kunkel G, Renz H. Modified T-cell activation pattern during specific immunotherapy (SIT) in cat-allergic patients. Clinical and Experimental Allergy 1999;29:618-25.

3. Daser A, Meissner N, Herz U, Renz $\mathrm{H}$. Role and modulation of T-cell cytokines in allergy. Current Opinion in Immunology 1995;7:762-70.

4. Braun A, Appel E, Baruch R, Herz U, Botchkarev V, Paus R, Brodie C, Renz $\mathrm{H}$. Role of nerve growth factor in a mouse model of allergic airway inflammation and asthma. Eur. J. Immunol 1998;28:3240-51.

5. Renz H. Soluble interleukin-4 receptor (sIL-4R) in allergic diseases. Inflammation Research 1999;48:425-31.

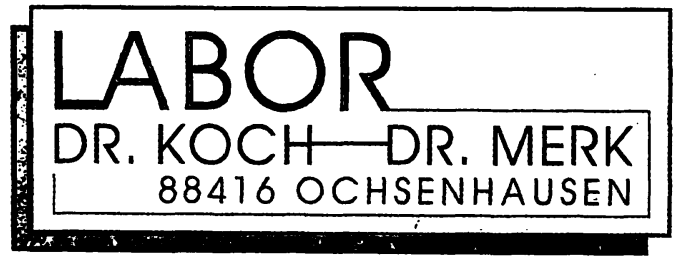




\section{Posterpreis „Molekulare und zelluläre Diagnostik“}

\begin{tabular}{|c|c|c|c|}
\hline C-reactive Protein (CRP) in Atherogenesis \\
Institute for Clinical Chemistry and Laboratory Medicine, University of Regensburg, Germany
\end{tabular}

\section{Fxtended Concept of Atherogenesis}

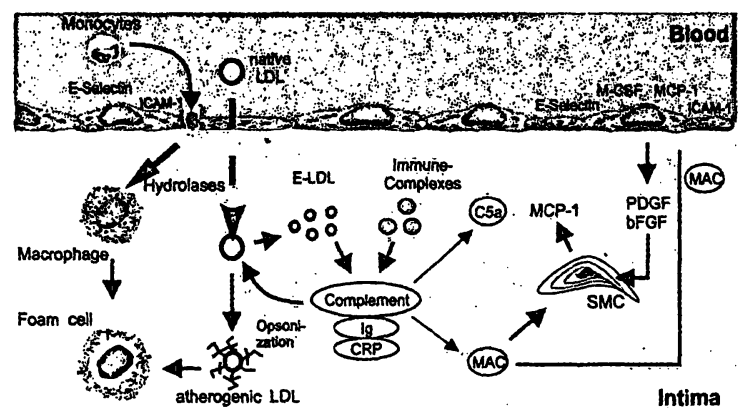

Subendothelial deposition of native LDL

$\square$ Modification of LDL by hydrolases (e. g. enzymatically modified LDL, E-LDL)

- Opsonization of modified LDL by innate (CRP, complement) and specific (immunoglobulins) opsonins

- Cellular uptake of opsonized LDL by macrophages and foam cell formation

$\square$ Complement activation by e. g. CRP, E-LDL and sublytic complement attack on smooth muscle cells (SMCs)

\section{MaterialsandiMëthods stown}

\section{Cells}

- Heparinized peripheral blood samples (PB) from healthy donors

a Heparinized PB from septicemia patients

- Elutriated monocytes cultured in serum free medium (Macrophage-SFM, Gibco Life Technologies), supplemented with human recombinant M-CSF

\section{Enzymatic Modification of LDL (E-LDL)}

a Triple enzyme modification of LDL (Bhakdi et al., J Exp Med 1995; 182: 1959): trypsin, cholesterol esterase, neuraminidase

\section{Flow-Cytometric Analysis}

a Whole blood staining of surface antigens (CD14, CD16, CD32, CD64)

- Detection of CRP binding by a polyclonal anti-CRP antibody.(CRPpAb)

a Detection of the CRP-R by a monoclonal antibody (clone RC10.2, CRP-RmAb)

- Four colour analysis on a FACSCalibur flow cytometer

\footnotetext{
"Presented at the Congress "Molecular and Cellular Diagnostics",
} October 3-5, 1999 in Regensburg, Germany.

\section{Af}

\section{Background}

CRP is emerging as a key mediator (complement activation, lipoprotein opsonization, monocyte chemotaxis) of inflammatory processes already in early atherogenesis. The identification and functional characterization of receptors for CRP have been the target of extensive research. Potential candidates are the FCyRI (CD64), the FCyRII (CD32), and a 'unique' CRP receptor (CRP-R).

\section{Goals}

Analysis of peripheral blood and peripheral blood monocyte subpopulations of healthy donors and patients with acute phase reactions by flow cytometry using a mAb against the leukocyte CRP-R as well as a polyclonal anti-CRP antibody

a Assessment of CRP-binding during foam cell formation as induced by enzymatically (trypsin, cholesterol esterase, neuraminidase) modified LDL (E-LDL)

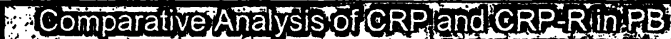

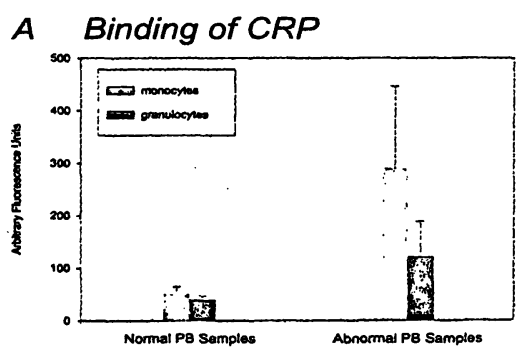

$B$ Expression of the CRP-R

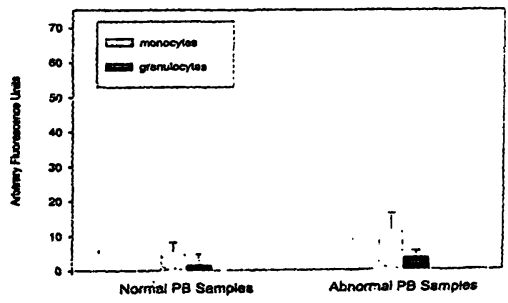

Analysis of CRP binding (A) and expression of the CRP-R $(B)$ in peripheral blood samples of healthy donors and patients with acute phase reactions. CRP binding and expression of the CRP-R on monocytes and granulocytes is enhanced under acute phase reactions. 
CRPand Peripheral Blood Monocyte Subpopulations
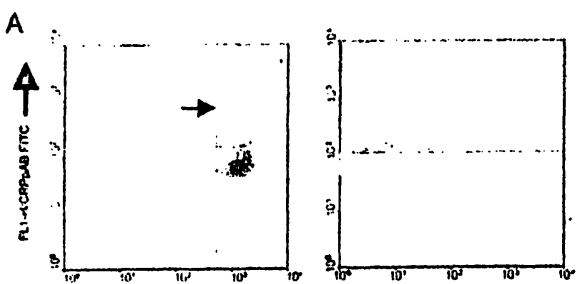

B
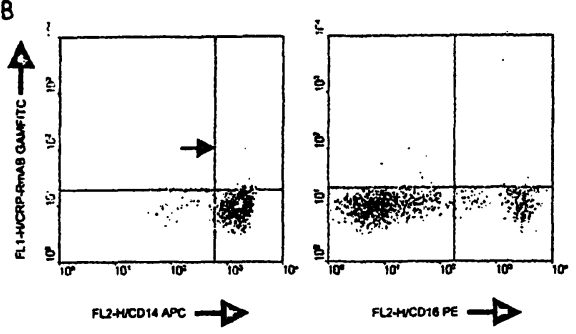

Analysis of CRP binding (A) and expression of the CRP-R (B) in peripheral blood monocyte subpopulations of patients with acute phase reactions. A distinct population of CD1 $14^{\text {sornt }}$ monocytes showed enhanced CRP binding and expression of the CRP-R (arrows)
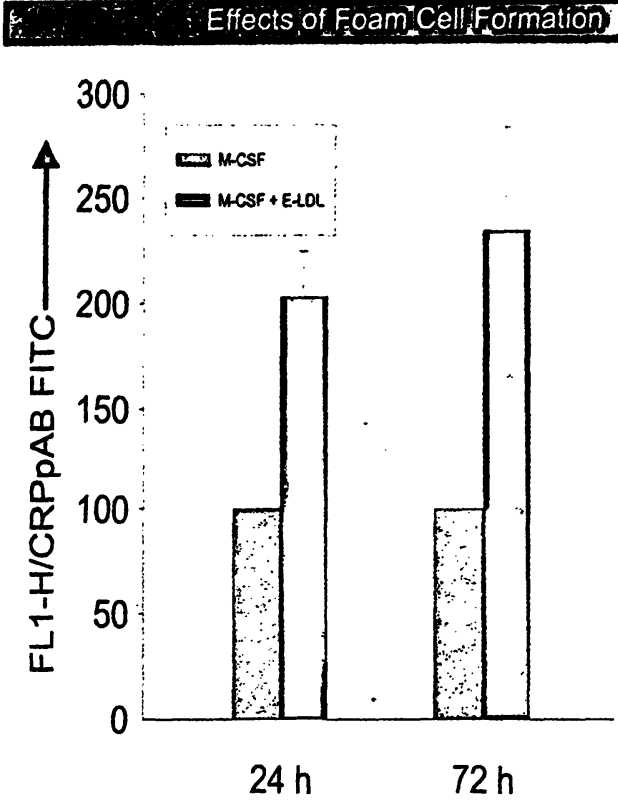

Analysis of CRP binding during lipid loading of freshly isolated monocytes ( $24 \mathrm{~h}$ and $72 \mathrm{~h}$, respectively). Binding of CRP is markedly enhanced on E-LDL loaded monocyte-derived macrophages compared to non loaded (MCSF $=100 \%$ ) cells already after $24 \mathrm{~h}$ incubation
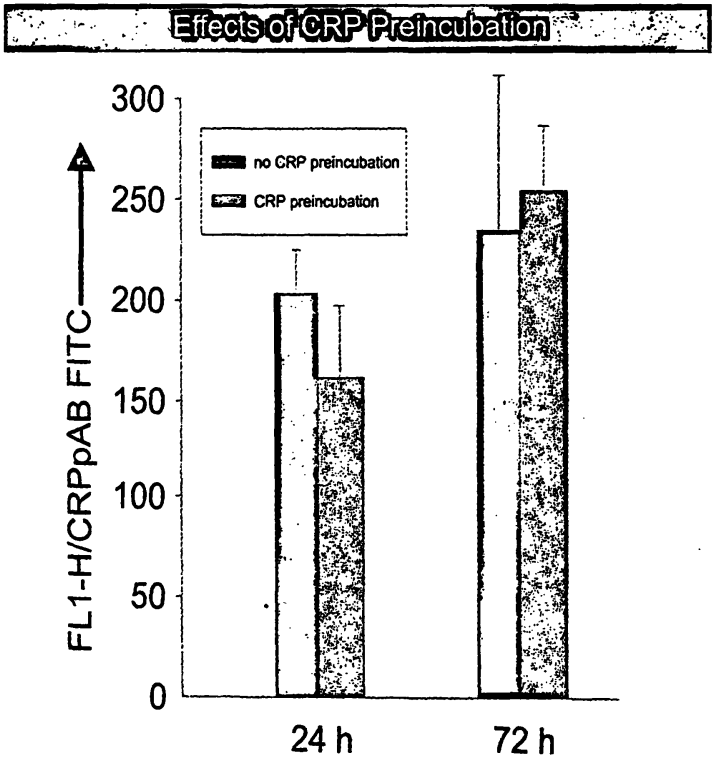

Analysis of CRP binding during lipid loading of freshly isolated monocytes with E-LDL ( $24 \mathrm{~h}$ and $72 \mathrm{~h}$, respectively) with and without CRP preincubation. CRP preincubation of loaded monocytederived macrophages does not influence significantly CRP binding

\section{\%}

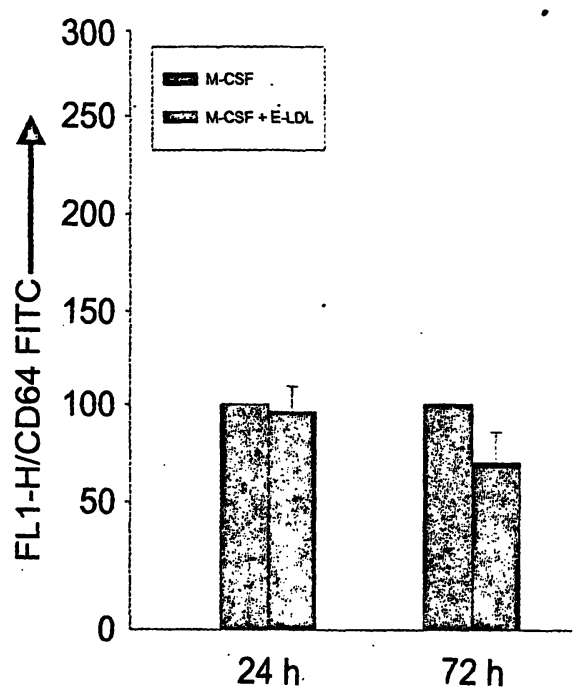

Expression of the ForRI/CD64 during lipid loading of freshly isolated monocytes ( $24 \mathrm{~h}$ and $72 \mathrm{~h}$, respectively) compared to non loaded (MCSF $=100 \%$ ) cells. Expression of the FCyRI/CD64 is not enhanced on E-LDL loaded monocyte-derived macrophages, even after $72 \mathrm{~h}$ incubation. 